\title{
SPITZER-IRS spectral fitting of discs around binary post-AGB stars (Corrigendum)
}

\author{
C. Gielen ${ }^{1, \star}$, H. Van Winckel ${ }^{1}$, M. Min ${ }^{16}$, L. B. F. M. Waters ${ }^{1,2}$, T. Lloyd Evans ${ }^{3}$, M. Matsuura ${ }^{4,5}$, P. Deroo ${ }^{6}$, \\ C. Dominik ${ }^{2,7}$, M. Reyniers ${ }^{8}$, A. Zijlstra ${ }^{9}$, K. D. Gordon ${ }^{10}$, F. Kemper ${ }^{9}$, R. Indebetouw ${ }^{11,15}$, M. Marengo ${ }^{12}$, \\ M. Meixner ${ }^{10}$, G. C. Sloan $^{13}$, A. G. G. M. Tielens ${ }^{14}$, and P. M. Woods ${ }^{9}$ \\ 1 Instituut voor Sterrenkunde, Katholieke Universiteit Leuven, Celestijnenlaan 200D, 3001 Leuven, Belgium \\ e-mail: clio.gielen@ster.kuleuven.be \\ 2 Sterrenkundig Instituut 'Anton Pannekoek', Universiteit Amsterdam, Kruislaan 403, 1098 Amsterdam, The Netherlands \\ 3 SUPA, School of Physics and Astronomy, University of St Andrews, North Haugh, St Andrews, Fife KY16 9SS, UK \\ ${ }^{4}$ UCL-Institute of Origins, Department of Physics and Astronomy, University College London, Gower Street, \\ London WC1E 6BT, UK \\ 5 UCL-Institute of Origins, Mullard Space Science Laboratory, University College London, Holmbury St. Mary, Dorking, \\ Surrey RH5 6NT, UK \\ 6 Jet Propulsion Laboratory, 4800 Oak Grove Drive, Pasadena, CA 91109, US \\ 7 Department of Astrophysics, Radboud University Nijmegen, PO Box 9010, 6500 GL Nijmegen, The Netherlands \\ 8 The Royal Meteorological Institute of Belgium, Department Observations, Ringlaan 3, 1180 Brussels, Belgium \\ 9 Jodrell Bank Centre for Astrophysics, Alan Turing Building, University of Manchester, Oxford Road, Manchester, M13 9PL, UK \\ 10 Space Telescope Science Institute, 3700 San Martin Drive, Baltimore, MD 21218, USA \\ 11 Department of Astronomy, University of Virginia, PO Box 3818, Charlottesville, VA 22903-0818, USA \\ 12 Harvard-Smithsonian Center for Astrophysics, 60 Garden Street, MS 65, Cambridge, MA 02138-1516, USA \\ 13 Department of Astronomy, Cornell University, Ithaca, NY 14853-6801, USA \\ 14 Leiden Observatory, J.H. Oort Building, Niels Bohrweg 2, 2333 CA Leiden, The Netherlands \\ 15 National Radio Astronomy Observatory, 520 Edgemont Road, Charlottesville, VA 22906, USA \\ 16 Astronomisch Instituut Utrecht, Universiteit Utrecht, Princetonplein 5, 3584 CC Utrecht, The Netherlands
}

A\&A, 490, 725-735 (2008), DOI: 10.1051/0004-6361:200810053

A\&A, 503, 843-854 (2009), DOI: 10.1051/0004-6361/200912060

A\&A, 508, 1391-1402 (2009), DOI: 10.1051/0004-6361/200912982

Key words. stars: abundances - stars: AGB and post-AGB - circumstellar matter - binaries: general - Magellanic Clouds errata, addenda

Recently, we have discovered an error in our Monte-Carlo spectral fitting routine, more specifically where the errors on the fluxes were rescaled to get a reduced $\chi^{2}$ of 1 . The rescaled errors were too big, resulting in too wide a range of "good" fits in our 100 step Monte-Carlo routine.

This problem affects Figs. 7-9 and Tables A.1, A.2 in Gielen et al. (2008), Table 3 in Gielen et al. (2009a), and Table 4 in Gielen et al. (2009b).

We corrected for this error and present the new values and errors in the tables below. The new values and errors nearly all fall within the old error range. Our best $\chi^{2}$ values and overall former scientific results are not affected. With these new errors some possible new trends in the dust parameters might be observed. These will be discussed in an upcoming paper where we extend the sample presented in Gielen et al. (2008) with newly obtained SPITZER-IRS data.

\footnotetext{
* Postdoctoral Fellow of the Fund for Scientific Research, Flanders.
}

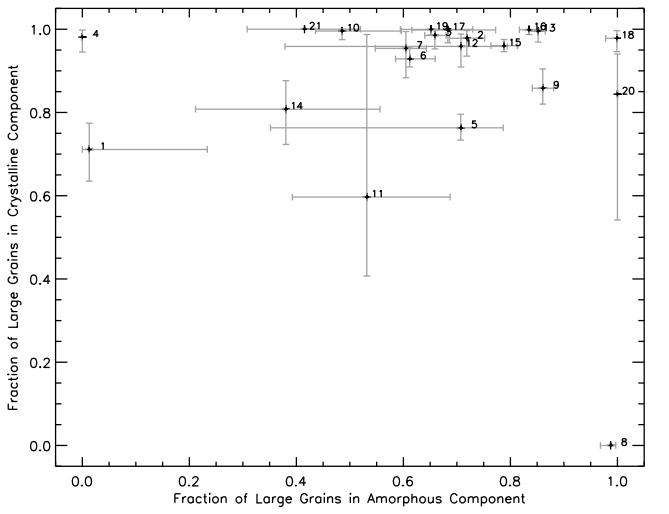

Fig. 1. Erratum for Fig. 7 in Gielen et al. (2008): the fraction of large grains in the amorphous component versus the fraction of large grains in the crystalline component, using the fitting with grain sizes of $0.1 \mu \mathrm{m}$ and $2.0 \mu \mathrm{m}$. Crystalline grains are almost completely made up of large $2.0 \mu \mathrm{m}$ grains. 
Table 1. Erratum for Table A.1 in Gielen et al. (2008): best-fit parameters deduced from our full spectral fitting.

\begin{tabular}{|c|c|c|c|c|c|c|c|c|}
\hline $\mathrm{N}^{\circ}$ & Name & $x^{2}$ & $\begin{array}{l}T_{\text {dust1 }} \\
(\mathrm{K}) \\
\end{array}$ & $\begin{array}{l}T_{\text {dust2 }} \\
(\mathrm{K})\end{array}$ & $\begin{array}{l}\text { Fraction } \\
T_{\text {dust } 1}-T_{\text {dust } 2} \\
\end{array}$ & $\begin{array}{l}T_{\text {cont1 }} \\
(\mathrm{K})\end{array}$ & $\begin{array}{l}T_{\text {cont2 }} \\
(\mathrm{K}) \\
\end{array}$ & $\begin{array}{l}\text { Fraction } \\
T_{\text {cont } 1}-T_{\text {cont } 2} \\
\end{array}$ \\
\hline 1 & EP Lyr & 56.7 & $100_{50}^{50}$ & $200_{50}^{50}$ & $0.90_{0.10}^{0.05}-0.10_{0.05}^{0.10}$ & $200_{50}^{50}$ & $994_{103}^{50}$ & $0.98_{0.01}^{0.01}-0.02_{0.01}^{0.01}$ \\
\hline 2 & HD 131356 & 3.5 & $200_{50}^{50}$ & $1000_{50}^{50}$ & $0.90_{0.05}^{0.05}-0.10_{0.05}^{0.05}$ & $200_{50}^{50}$ & $500_{50}^{50}$ & $0.90_{0.01}^{0.01}-0.10_{0.01}^{0.01}$ \\
\hline 3 & HD 213985 & 4.4 & $184_{87}^{50}$ & $1000_{50}^{50}$ & $0.90_{0.05}^{0.05}-0.10_{0.05}^{0.05}$ & $200_{50}^{50}$ & $884_{87}^{50}$ & $0.98_{0.01}^{0.01}-0.02_{0.01}^{0.01}$ \\
\hline 4 & HD 52961 & 72.2 & $200_{50}^{50}$ & $800_{50}^{50}$ & $0.90_{0.05}^{0.05}-0.10_{0.05}^{0.05}$ & $100_{50}^{50}$ & $1000_{50}^{50}$ & $0.99_{0.01}^{0.01}-0.01_{0.01}^{0.01}$ \\
\hline 5 & IRAS 05208 & 4.5 & $292_{95}^{50}$ & $923_{113}^{78}$ & $0.80_{0.10}^{0.05}-0.20_{0.05}^{0.10}$ & $200_{50}^{50}$ & $400_{50}^{50}$ & $0.85_{0.01}^{0.01}-0.15_{0.01}^{0.01}$ \\
\hline 6 & IRAS 09060 & 3.6 & $200_{50}^{50}$ & $728_{130}^{73}$ & $0.90_{0.05}^{0.05}-0.10_{0.05}^{0.05}$ & $228_{130}^{73}$ & $834_{237}^{141}$ & $0.93_{0.02}^{0.02}-0.07_{0.02}^{0.02}$ \\
\hline 7 & IRAS 09144 & 6.1 & $200_{50}^{50}$ & $504_{50}^{111}$ & $0.90_{0.05}^{0.05}-0.10_{0.05}^{0.05}$ & $200_{50}^{50}$ & $796_{111}^{50}$ & $0.94_{0.01}^{0.01}-0.06_{0.01}^{0.01}$ \\
\hline 8 & IRAS 10174 & 13.9 & $300_{50}^{50}$ & $400_{50}^{50}$ & $0.90_{0.05}^{0.05}-0.10_{0.05}^{0.05}$ & $100_{50}^{50}$ & $300_{50}^{50}$ & $0.97_{0.01}^{0.01}-0.03_{0.01}^{0.01}$ \\
\hline 9 & IRAS 16230 & 4.9 & $200_{50}^{50}$ & $500_{50}^{50}$ & $0.90_{0.05}^{0.05}-0.10_{0.05}^{0.05}$ & $100_{50}^{50}$ & $500_{50}^{50}$ & $0.95_{0.05}^{0.05}-0.05_{0.05}^{0.05}$ \\
\hline 10 & IRAS 17038 & 2.9 & $317_{50}^{85}$ & $871_{80}^{60}$ & $0.80_{0.10}^{0.10}-0.20_{0.10}^{0.10}$ & $200_{50}^{50}$ & $591_{96}^{50}$ & $0.97_{0.02}^{0.01}-0.03_{0.01}^{0.02}$ \\
\hline 11 & IRAS 17243 & 2.3 & $200_{50}^{50}$ & $486_{89}^{50}$ & $0.90_{0.10}^{0.05}-0.10_{0.05}^{0.10}$ & $200_{50}^{50}$ & $600_{50}^{50}$ & $0.90_{0.01}^{0.01}-0.10_{0.01}^{0.01}$ \\
\hline 12 & IRAS 19125 & 3.9 & $100_{50}^{50}$ & $200_{50}^{50}$ & $0.90_{0.05}^{0.05}-0.10_{0.05}^{0.05}$ & $482_{309}^{50}$ & $788_{206}^{50}$ & $0.86_{0.05}^{0.01}-0.14_{0.01}^{0.01}$ \\
\hline 13 & IRAS 19157 & 5.5 & $200_{50}^{50}$ & $695_{106}^{50}$ & $0.90_{0.05}^{0.05}-0.10_{0.05}^{0.05}$ & $200_{50}^{50}$ & $705_{50}^{106}$ & $0.96_{0.01}^{0.01}-0.04_{0.01}^{0.01}$ \\
\hline 14 & IRAS 20056 & 3.8 & $100_{50}^{50}$ & $200_{50}^{50}$ & $0.10_{0.10}^{0.20}-0.90_{0.20}^{0.10}$ & $200_{50}^{50}$ & $600_{50}^{50}$ & $0.88_{0.01}^{0.01}-0.12_{0.01}^{0.01}$ \\
\hline 15 & RU Cen & 3.4 & $287_{91}^{50}$ & $575_{176}^{50}$ & $0.90_{0.30}^{0.05}-0.10_{0.05}^{0.30}$ & $200_{50}^{50}$ & $599_{50}^{50}$ & $0.99_{0.01}^{0.01}-0.01_{0.01}^{0.01}$ \\
\hline 16 & SAO 173329 & 3.1 & $200_{50}^{50}$ & $702_{50}^{139}$ & $0.90_{0.05}^{0.05}-0.10_{0.05}^{0.05}$ & $200_{50}^{50}$ & $600_{50}^{50}$ & $0.93_{0.01}^{0.01}-0.07_{0.01}^{0.01}$ \\
\hline 17 & ST Pup & 8.4 & $200_{50}^{50}$ & $500_{50}^{50}$ & $0.90_{0.05}^{0.05}-0.10_{0.05}^{0.05}$ & $200_{50}^{50}$ & $500_{50}^{50}$ & $0.95_{0.01}^{0.01}-0.05_{0.01}^{0.01}$ \\
\hline 18 & SU Gem & 1.8 & $154_{54}^{105}$ & $558_{59}^{96}$ & $0.80_{0.10}^{0.10}-0.20_{0.10}^{0.10}$ & $200_{50}^{50}$ & $800_{50}^{50}$ & $0.95_{0.01}^{0.01}-0.05_{0.01}^{0.01}$ \\
\hline 19 & SX Cen & 4.3 & $257_{58}^{50}$ & $968_{69}^{50}$ & $0.80_{0.10}^{0.10}-0.20_{0.10}^{0.10}$ & $200_{50}^{50}$ & $691_{96}^{50}$ & $0.94_{0.01}^{0.01}-0.06_{0.01}^{0.01}$ \\
\hline 20 & TW Cam & 2.3 & $261_{62}^{50}$ & $400_{50}^{50}$ & $0.60_{0.05}^{0.10}-0.40_{0.10}^{0.05}$ & $100_{50}^{50}$ & $500_{50}^{50}$ & $0.95_{0.01}^{0.01}-0.05_{0.01}^{0.01}$ \\
\hline 21 & UY CMa & 2.9 & $200_{50}^{52}$ & $726_{50}^{50}$ & $0.90_{0.05}^{0.05}-0.10_{0.05}^{0.05}$ & $200_{50}^{50}$ & $500_{50}^{50}$ & $0.83_{0.01}^{0.01}-0.17_{0.01}^{0.01}$ \\
\hline
\end{tabular}

Notes. Listed are the $\chi^{2}$, dust and continuum temperatures and their relative fractions.

Table 2. Erratum for Table A.2 in Gielen et al. (2008): best-fit parameters deduced from our full spectral fitting.

\begin{tabular}{|c|c|c|c|c|c|c|}
\hline $\mathrm{N}^{\circ}$ & Name & $\begin{array}{c}\text { Olivine } \\
\text { small-large }\end{array}$ & $\begin{array}{l}\text { Pyroxene } \\
\text { small-large }\end{array}$ & $\begin{array}{c}\text { Forsterite } \\
\text { small-large }\end{array}$ & $\begin{array}{c}\text { Enstatite } \\
\text { small-large }\end{array}$ & Continuum \\
\hline 1 & EP Lyr & $0.00_{0.00}^{0.00}-0.00_{0.00}^{0.00}$ & $15.36_{9.25}^{12.96}-61.34_{23.01}^{10.71}$ & $14.43_{2.17}^{4.61}-0.02_{0.02}^{0.00}$ & $0.00_{0.00}^{0.00}-8.85_{3.90}^{4.49}$ & $53.51_{1.43}^{3.36}$ \\
\hline 2 & HD 131356 & $0.00_{0.00}^{0.00}-30.48_{1.44}^{1.50}$ & $2.87_{1.77}^{1.79}-53.91_{2.72}^{2.57}$ & $12.06_{0.62}^{0.65}-0.03_{0.03}^{1.00}$ & $0.00_{0.00}^{0.00}-0.65_{0.56}^{1.3 .30}$ & $76.34_{0.24}^{0.30}$ \\
\hline 3 & HD 213985 & $0.00_{0.00}^{0.00}-36.08_{2.21}^{2.20}$ & $12.58_{9.92}^{3.42}-29.27_{6.99}^{25.84}$ & $8.02_{1.63}^{1.79}-7.74_{4.86}^{3.26}$ & $0.00_{0.00}^{0.00}-6.31_{4.45}^{2.09}$ & $76.21_{1.51}^{0.57}$ \\
\hline 4 & HD 52961 & $0.00_{0.00}^{0.00}-0.00_{0.00}^{0.00}$ & $70.65_{1.93}^{1.61}-0.00_{0.00}^{0.009}$ & $20.84_{3.57}^{3.81}-8.40_{4.94}^{5.18}$ & $0.00_{0.00}^{0.00}-0.12_{0.12}^{2.07}$ & $65.66_{0.62}^{0.57}$ \\
\hline 5 & IRAS 05208 & $0.00_{0.00}^{0.00}-9.52_{2.42}^{2.40}$ & $33.16_{1.29}^{1.52}-0.00_{0.00}^{0.00}$ & $25.76_{1.10}^{1.34}-0.00_{0.00}^{0.00}$ & $2.10_{1.67}^{2.13}-29.47_{3.27}^{2.80}$ & $69.06_{0.38}^{0.32}$ \\
\hline 6 & IRAS 09060 & $0.06_{0.06}^{1.80}-32.70_{4.58}^{4.46}$ & $39.74_{2.66}^{3.00}-0.48_{0.49}^{4.21}$ & $14.95_{1.81}^{1.37}-1.11_{1.11}^{2.90}$ & $0.01_{0.01}^{1.010}-10.95_{2.51}^{3.37}$ & $72.43_{2.20}^{1.24}$ \\
\hline 7 & IRAS 09144 & $0.00_{0.00}^{0.00}-39.21_{4.59}^{2.04}$ & $17.77_{2.65}^{1.54}-34.88_{3.03}^{7.04}$ & $7.99_{0.63}^{0.75}-0.00_{0.00}^{0.00}$ & $0.00_{0.00}^{0.00}-0.14_{0.14}^{1.21}$ & $72.51_{0.30}^{0.39}$ \\
\hline 8 & IRAS 10174 & $8.70_{3.38}^{4.93}-39.99_{6.00}^{4.24}$ & $26.10_{3.10}^{2.29}-25.21_{3.46}^{4.19}$ & $0.00_{0.00}^{0.00}-0.00_{0.00}^{0.00}$ & $0.00_{0.00}^{0.00}-0.00_{0.00}^{0.00}$ & $31.48_{0.49}^{0.54}$ \\
\hline 9 & IRAS 16230 & $0.00_{0.00}^{0.00}-47.54_{2.30}^{0.35}$ & $0.00_{0.00}^{0.00}-29.46_{2.39}^{2.01}$ & $18.20_{0.98}^{0.90}-4.23_{1.50}^{1.38}$ & $0.00_{0.00}^{0.00}-0.58_{0.54}^{1.51}$ & $75.74_{0.25}^{0.29}$ \\
\hline 10 & IRAS 17038 & $0.00_{0.00}^{0.00}-31.29_{2.85}^{2.25}$ & $0.03_{0.03}^{0.92}-31.02_{2.54}^{4.33}$ & $21.65_{0.91}^{0.77}-0.00_{0.00}^{0.00}$ & $0.00_{0.00}^{0.00}-16.00_{2.13}^{1.89}$ & $81.71_{0.35}^{0.23}$ \\
\hline 11 & IRAS 17243 & $0.49_{0.49}^{7.03}-42.74_{2.29}^{2.73}$ & $20.13_{3.29}^{10.90}-14.61_{9.18}^{5.25}$ & $17.34_{0.95}^{0.90}-0.00_{0.00}^{0.00}$ & $0.00_{0.00}^{0.00}-4.69_{2.60}^{1.76}$ & $83.22_{0.29}^{0.41}$ \\
\hline 12 & IRAS 19125 & $8.22_{6.32}^{7.72}-6.24_{4.96}^{6.47}$ & $8.66_{4.28}^{8.00}-45.53_{20.03}^{5.92}$ & $7.98_{0.74}^{0.98}-7.87_{1.22}^{1.34}$ & $0.00_{0.00}^{0.00}-15.50_{1.34}^{2.60}$ & $69.72_{0.44}^{7.00}$ \\
\hline 13 & IRAS 19157 & $0.00_{0.00}^{0.00}-63.21_{3.12}^{5.30}$ & $8.43_{3.41}^{4.28}-12.05_{8.01}^{6.89}$ & $14.58_{1.49}^{1.36}-0.02_{0.02}^{0.94}$ & $0.00_{0.00}^{0.00}-1.70_{1.35}^{2.00}$ & $82.28_{0.53}^{0.61}$ \\
\hline 14 & IRAS 20056 & $0.45_{0.45}^{2.96}-31.83_{3.94}^{3.62}$ & $34.60_{2.66}^{2.35}-0.30_{0.30}^{5.33}$ & $15.86_{1.10}^{1.04}-0.02_{0.02}^{0.85}$ & $0.00_{0.00}^{0.00}-16.93_{1.77}^{1.80}$ & $83.48_{0.64}^{0.20}$ \\
\hline 15 & RU Cen & $0.00_{0.00}^{0.00}-27.92_{2.49}^{3.174}$ & $1.24_{1.06}^{2.06}-27.81_{3.95}^{3.80}$ & $28.63_{1.80}^{1.83}-3.34_{2.68}^{2.71}$ & $0.00_{0.00}^{0.00}-11.06_{2.11}^{1.71}$ & $80.62_{0.76}^{0.34}$ \\
\hline 16 & SAO 173329 & $0.00_{000}^{0.00}-52.55_{2.35}^{1.74}$ & $0.02_{0.02}^{0.50}-28.64_{247}^{2.15}$ & $9.21_{0.09}^{1.09}-0.02_{0.02}^{0.00}$ & $0.00_{0.00}^{0.00}-9.56_{185}^{2.63}$ & $82.54_{0.20}^{0.16}$ \\
\hline 17 & ST Pup & $0.00_{0.00}^{0.00}-32.87_{1.23}^{1.29}$ & $0.71_{0.66}^{1.86}-51.93_{3.17}^{1.79}$ & $13.19_{0.46}^{0.57}-0.03_{0.03}^{0.83}$ & $0.00_{0.00}^{0.00}-1.27_{0.90}^{1.1 .16}$ & $54.60_{0.37}^{0.53}$ \\
\hline 18 & SU Gem & $0.00_{0.00}^{0.00}-58.69_{3.77}^{3.60}$ & $0.70_{0.69}^{2.86}-12.03_{5.11}^{3.93}$ & $23.41_{1.75}^{2.31}-2.30_{1.96}^{3.69}$ & $0.00_{0.00}^{0.00}-2.87_{2.11}^{0.00}$ & $88.40_{0.43}^{0.47}$ \\
\hline 19 & SX Cen & $0.00_{0.00}^{0.00}-48.37_{4.30}^{3.30}$ & $9.19_{5.82}^{11.44}-23.70_{2367}^{12.70}$ & $10.82_{1.97}^{3.64}-0.53^{4.29}$ & $0.00_{0.00}^{0.00}-7.39_{3.15}^{4.69}$ & $76.16_{116}^{1.50}$ \\
\hline 20 & TW Cam & $0.00_{0.00}^{0.00}-84.42_{2.04}^{1.81}$ & $0.00_{0.00}^{0.00}-0.00_{0.00}^{0.00}$ & $15.20_{1.73}^{1.47}-0.00_{0.00}^{0.00}$ & $0.00_{0.00}^{0.00}-0.37_{0.37}^{1.84}$ & $90.63_{0.18}^{0.10}$ \\
\hline 21 & UY CMa & $0.00_{0.00}^{0.00}-15.55_{1.96}^{2.34}$ & $2.54_{2.02}^{2.53}-58.59_{3.68}^{3.01}$ & $16.18_{1.78}^{2.50}-4.48_{3.42}^{2.93}$ & $0.00_{0.00}^{0.00}-2.65_{1.43}^{1.53}$ & $78.14_{0.42}^{0.18}$ \\
\hline
\end{tabular}

Notes. The abundances of small $(2.0 \mu \mathrm{m})$ and large $(4.0 \mu \mathrm{m})$ grains of the various dust species are given as fractions of the total mass, excluding the dust responsible for the continuum emission. The last column gives the continuum flux contribution, listed as a percentage of the total integrated flux over the full wavelength range. 
C. Gielen et al.: SPITZER-IRS spectral fitting of discs around binary post-AGB stars

Table 3. Erratum for Table 3 in Gielen et al. (2009a): best-fit parameters deduced from our full spectral fitting.

\begin{tabular}{|c|c|c|c|c|c|c|c|c|c|c|c|}
\hline & \multicolumn{2}{|c|}{ Name } & $x^{2}$ & $\begin{array}{l}T_{\text {dust1 }} \\
(\mathrm{K})\end{array}$ & $\begin{array}{l}T_{\text {dust2 }} \\
(\mathrm{K})\end{array}$ & \multicolumn{2}{|c|}{$\begin{array}{l}\text { Fraction } \\
T_{\text {dust } 1}-T_{\text {dust2 }}\end{array}$} & $\begin{array}{l}T_{\text {cont } 1} \\
(\mathrm{~K})\end{array}$ & $\begin{array}{l}T_{\text {cont } 2} \\
(\mathrm{~K})\end{array}$ & \multicolumn{2}{|c|}{$\begin{array}{l}\text { Fraction } \\
T_{\text {cont1 }}-T_{\text {cont2 }}\end{array}$} \\
\hline & \multicolumn{2}{|c|}{$\begin{array}{l}\text { EP Lyr } \\
\text { HD } 52961\end{array}$} & $\begin{array}{r}5.4 \\
50.0\end{array}$ & $\begin{array}{l}100_{50}^{50} \\
200_{50}^{50}\end{array}$ & $\begin{array}{l}200_{50}^{50} \\
700_{50}^{50}\end{array}$ & $\begin{array}{l}0.90_{0.05}^{0.10} \\
0.90_{0.05}^{0.05}\end{array}$ & $\begin{array}{l}-0.10_{0.10}^{0.05} \\
-0.10_{0.05}^{0.05}\end{array}$ & $\begin{array}{l}100_{50}^{50} \\
100_{50}^{50}\end{array}$ & $\begin{array}{l}643_{50}^{302} \\
1000_{50}^{50}\end{array}$ & \multicolumn{2}{|c|}{$\begin{array}{l}0.98_{0.04}^{0.01}-0.02_{0.01}^{0.04} \\
0.99_{0.01}^{0.01}-0.01_{0.01}^{0.01}\end{array}$} \\
\hline \multicolumn{2}{|l|}{ Name } & \multicolumn{3}{|c|}{$\begin{array}{c}\text { Olivine } \\
\text { small-large }\end{array}$} & \multicolumn{2}{|c|}{$\begin{array}{l}\text { Pyroxene } \\
\text { small-large }\end{array}$} & \multicolumn{2}{|c|}{$\begin{array}{c}\text { Forsterite } \\
\text { small-large }\end{array}$} & \multicolumn{2}{|c|}{$\begin{array}{c}\text { Enstatite } \\
\text { small-large }\end{array}$} & Continuum \\
\hline \multicolumn{2}{|c|}{$\begin{array}{l}\text { EP Lyr } \\
\text { HD } 52961\end{array}$} & \multicolumn{3}{|c|}{$\begin{array}{c}0.24_{0.24}^{16.83}-8.74_{7.64}^{7.92} \\
0.00_{0.00}^{0.00}-0.00_{0.00}^{0.00}\end{array}$} & \multicolumn{2}{|c|}{$\begin{array}{l}7.17_{4.79}^{13.69}-8.09_{7.24}^{12.67} \\
59.17_{0.69}^{0.72}-0.00_{0.00}^{0.00}\end{array}$} & \multicolumn{2}{|c|}{$\begin{array}{l}35.18_{2.78}^{3.04}-2.08_{1.89}^{2.61} \\
0.77_{0.69}^{1.46}-40.06_{1.62}^{1.02}\end{array}$} & \multicolumn{2}{|c|}{$\begin{array}{c}0.00_{0.00}^{0.00}-38.50_{3.46}^{4.30} \\
0.00_{0.00}^{0.00}-0.00_{0.00}^{0.00}\end{array}$} & $\begin{array}{l}57.99_{3.60}^{2.53} \\
68.88_{0.46}^{0.42}\end{array}$ \\
\hline
\end{tabular}

Notes. Listed are the $\chi^{2}$, dust, and continuum temperatures and their relative fractions. Best-fit parameters deduced from our full spectral fitting. The abundances of small and large grains of the various dust species are given as fractions of the total mass, excluding the dust responsible for the continuum emission. The last column gives the continuum flux contribution, listed as a percentage of the total integrated flux over the full wavelength range.

Table 4. Erratum for Table 4 in Gielen et al. (2009b): best-fit parameters deduced from our full spectral fitting.

\begin{tabular}{|c|c|c|c|c|c|c|c|c|c|c|c|}
\hline & Name & & $\chi^{2}$ & $\begin{array}{l}T_{\text {dust1 }} \\
(\mathrm{K})\end{array}$ & $\begin{array}{l}T_{\text {dust2 }} \\
(\mathrm{K})\end{array}$ & \multicolumn{2}{|c|}{$\begin{array}{l}\text { Fraction } \\
T_{\text {dust } 1-T_{\text {dust2 }}}\end{array}$} & $\begin{array}{l}T_{\text {cont1 }} \\
(\mathrm{K})\end{array}$ & $\begin{array}{l}T_{\text {cont2 }} \\
(\mathrm{K})\end{array}$ & \multicolumn{2}{|l|}{$\begin{array}{l}\text { Fraction } \\
T_{\text {cont } 1-T_{\text {cont2 }}} \\
\end{array}$} \\
\hline & $\begin{array}{l}\text { MACHO } 79.55 \\
\text { MACHO } 82.84\end{array}$ & $\begin{array}{l}01.13 \\
05.15\end{array}$ & $\begin{array}{l}5.1 \\
3.9\end{array}$ & $\begin{array}{l}200_{50}^{50} \\
200_{50}^{50}\end{array}$ & $\begin{array}{l}725_{50}^{83} \\
519_{75}^{82}\end{array}$ & \multicolumn{2}{|c|}{$\begin{array}{l}0.90_{0.05}^{0.05}-0.10_{0.05}^{0.05} \\
0.90_{0.05}^{0.05}-0.10_{0.05}^{0.05}\end{array}$} & $\begin{array}{l}346_{246}^{184} \\
300_{50}^{50}\end{array}$ & $\begin{array}{l}623_{50}^{99} \\
500_{50}^{50}\end{array}$ & \multicolumn{2}{|c|}{$\begin{array}{l}0.21_{0.18}^{0.69}-0.79_{0.69}^{0.18} \\
0.82_{0.02}^{0.03}-0.18_{0.03}^{0.02}\end{array}$} \\
\hline Name & & \multicolumn{3}{|c|}{$\begin{array}{c}\text { Olivine } \\
\text { small-large }\end{array}$} & \multicolumn{2}{|c|}{$\begin{array}{c}\text { Pyroxene } \\
\text { small-large }\end{array}$} & \multicolumn{2}{|c|}{$\begin{array}{c}\text { Forsterite } \\
\text { small-large }\end{array}$} & \multicolumn{2}{|c|}{$\begin{array}{c}\text { Enstatite } \\
\text { small-large }\end{array}$} & Continuum \\
\hline $\begin{array}{l}\text { MACH } \\
\text { MACH }\end{array}$ & $\begin{array}{l}\text { O } 79.5501 .13 \\
\text { O } 82.8405 .15\end{array}$ & \multicolumn{3}{|c|}{$\begin{array}{c}0.00_{0.00}^{0.00}-0.00_{0.00}^{0.00} \\
0.96_{0.95}^{7.13}-4.13_{3.97}^{10.91}\end{array}$} & \multicolumn{2}{|c|}{$\begin{array}{c}48.45_{7.00}^{4.75}-0.37_{0.37}^{20.55} \\
52.80_{9.36}^{10.75}-4.01_{3.92}^{18.56}\end{array}$} & \multicolumn{2}{|c|}{$\begin{array}{l}0.00_{0.00}^{0.00}-44.54_{3.32}^{3.47} \\
5.50_{2.26}^{2.76}-20.52_{7.06}^{6.47}\end{array}$} & \multicolumn{2}{|c|}{$\begin{array}{c}0.17_{0.17}^{2.86}-6.47_{4.25}^{5.76} \\
0.14_{0.14}^{3.66}-11.95_{5.83}^{5.93}\end{array}$} & $\begin{array}{l}89.27_{0.86}^{0.70} \\
82.63_{1.86}^{1.86}\end{array}$ \\
\hline
\end{tabular}

Notes. Listed are the $\chi^{2}$, dust, and continuum temperatures and their relative fractions. Best-fit parameters deduced from our full spectral fitting. The abundances of small $(0.1 \mu \mathrm{m})$ and large $(2.0 \mu \mathrm{m})$ grains of the various dust species are given as fractions of the total mass, excluding the dust responsible for the continuum emission. The last column gives the continuum flux contribution, listed as a percentage of the total integrated flux over the full wavelength range.

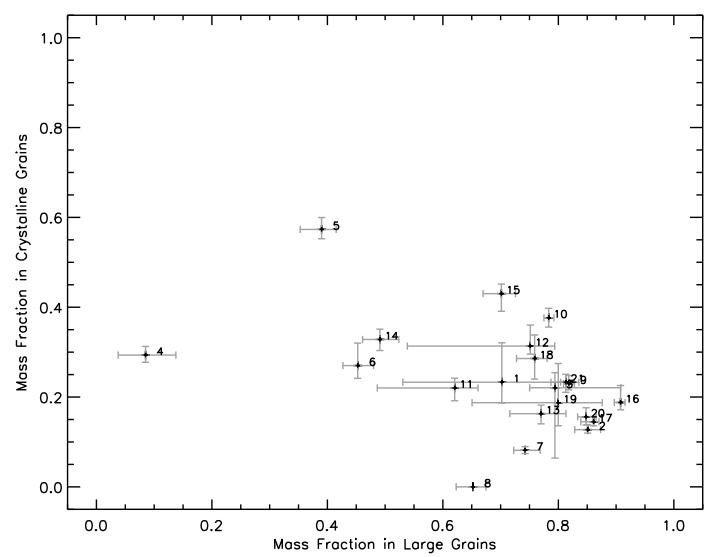

Fig. 2. Erratum for Fig. 8 in Gielen et al. (2008): the mass fraction in large grains $(4.0 \mu \mathrm{m})$ plotted against the mass fraction in crystalline grains, as derived from our best-fit parameters.

Acknowledgements. C.G. and H.V.W. acknowledge support of the Fund for Scientific Research of Flanders (FWO) under the grant G.0178.02. and G.0470.07. This work is based on observations made with the Spitzer Space Telescope, which is operated by the Jet Propulsion Laboratory, California Institute of Technology, under a contract with NASA.

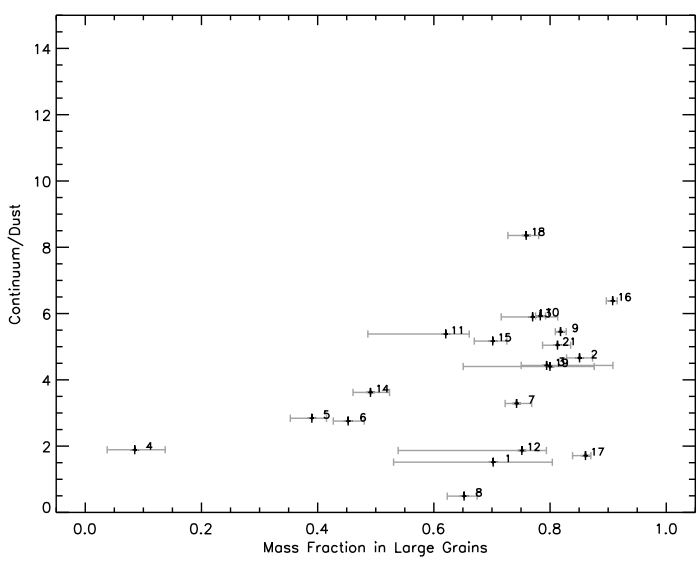

Fig. 3. Erratum for Fig. 9 in Gielen et al. (2008): the continuum-to-dust ratio of the observed spectra plotted against the mass fraction on large grains $(4.0 \mu \mathrm{m})$.

\section{References}

Gielen, C., Van Winckel, H., Min, M., Waters, L. B. F. M., \& Lloyd Evans, T. 2008, A\&A, 490, 725

Gielen, C., Van Winckel, H., Matsuura, M., et al. 2009a, A\&A, 503, 843

Gielen, C., Van Winckel, H., Reyniers, M., et al. 2009b, A\&A, 508, 1391 